packte Silphion gewcgen wird, während Matrosen unter Aufsicht eines Wächters die Säcke im unteren Schiffsraume verstauen.

Der englische Botaniker Falkoner hat im nördlichen Kaschmir ein hohes Doldengewächs gefunden, welches eine Art Asant liefert und von ihm als Narthex bestimmt ist.

Friedländer ${ }^{1}$ ) sagt: „Die Abbildung dieser Pflanze entspricht genau dem Bilde der Münzen .. a. s. w. Nach ihr ist der starke gerillte Stengel reichlich mit fiederspaltigen Blättern besetzt, deren Stiele aufgeblasene Scheiden bilden, an der Spitze des Stengels sitzt eine nicht reich verzweigte Dolde.

Ob und was für ein Zusammenhang zwischen der Pflanze Indien's und Kyrene's herrscht, läßt sich nicht entscheiden.

\title{
Ueber die Chrysophansäure.
}

Von O. A. Oesterle.

(Eingegangen den 8. VII. 1905.)

Chrysophansäure $^{2}$ ), die nach Liebermann und Fischer ${ }^{8}$ ) als Dioxymethylanthrachinon aufgefaßt werden $m u B$, ist in den unterirdischen Organen verschiedener Rheum- und Rumex-Arten, in den Sennesblättern und in der Rinde von Cassia bijuga Vogel ${ }^{4}$ ) (Fedegosa do mato virgem) nachgewiesen worden. Nach Limousin ${ }^{5}$ ), Aweng ${ }^{6}$ ) and Le Prince ${ }^{7}$ ) soll sie auch in der Rinde von Rhamnus Frangula und von Rhamnus Purshiana in Form eines Glykosides vorhanden sein, Angaben, die jedoch von $\mathrm{J}_{0} \mathrm{wett}^{8}$ ) bestritten werden.

Wie Liebermann und Siedler ${ }^{\theta}$ ) zeigten, entsteht Chrysophansăure bei der Oxydation des Chrysarobins in alkalischer Lösung.

1) Numismat. Zeitschr. 1872.

2) A. Brissemoret (Contribution à l'étude des purgatifs organiques, Paris 1903) und Tschirch (Festschrift Prof. Vogl) schlagen vor, die Bezeichnung Chrysophanol an Stelle von Chrysophansäure zu wählea.

B) Ber. d. d. chem. Ges. 8 (1875), 1104.

4) Peckolt, Arch. d. Pharm. 1868.

3) Journ. de Pharm. et de Chimie 1885, 80 .

6) Pharm. Zentralh. 1898, 776. Apoth.-Ztg. 1900, 15, 537; 1932, 17, 372.

7) Compt. rend. 1899, 129, 60.

8) Chemical Examination of Cascara bark. 1904, No. 47, of published papers from the Wellcome Chemical research laboratories.

9) Ber. d. d. chem. Ges. 11 (1878), 1606. 
Vergleicht man die Angaben, welche sich über die Chrysophansäure finden, so fällt vor allem auf, $\mathrm{daß}$ die Schmelzpunkte innerhalb weiter Grenzen schwanken. Es sind folgende Schmelzpunkte gefunden worden vou:

Warren de la Rue und Hugo Müller.

Kopp-Will, Jahresber. 1857. $516 ; 1862,323$

H. Grothe Kopp.Will, Jalıresber. 1863 707

\section{Skraup}

Wien akad. Ber. 70, 235;

Jahresber. 1874, 899

\section{Keussle}

Inaug - Ilissert. Riga 1879

Liebermann . . . Ann. 212 (1882), 37

\section{tirandis}

Jahresber. 1892, $160 \check{4}$

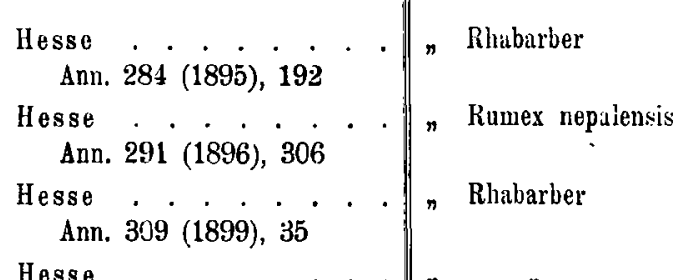

Hesse . . . . . . n n

Ann. 309 (1899), 36

Hesse . . . . . . " engl. Rhabarber

Ann. 309,48

Hesse . . . . . . Rumex obtusifol.

inn. 309,52

Hesse .. . . . . . „ Chrysarobin

Ann 309,61

Hunkel . . . . . . „ Rlabarber

Pharmaceutic. Archives Vol.3, No. 11

Tschirch und Hiepe . . . " Senna

Arch. d. Pharm. 1900, 43j

Tschirch und Heuberger . " Rhabarber

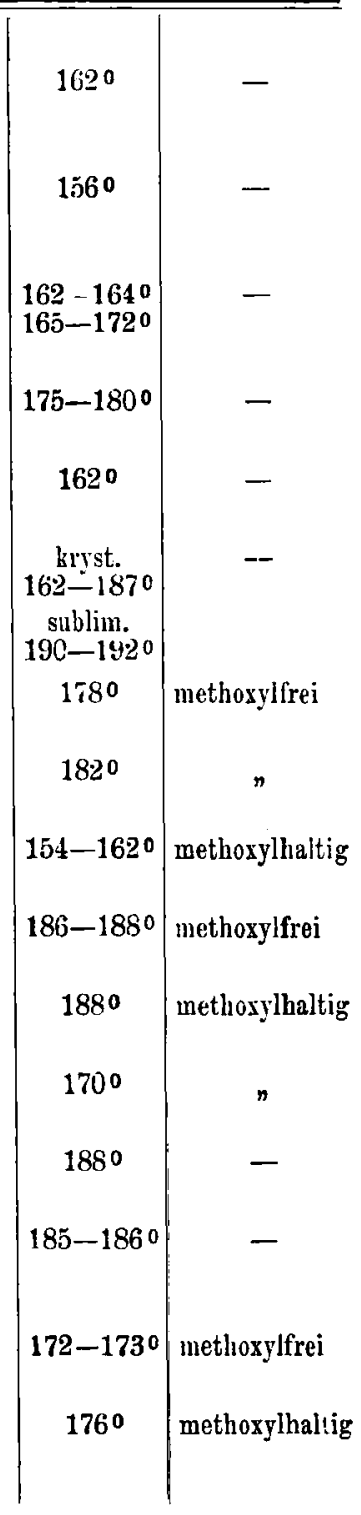

28* 


\begin{tabular}{|c|c|c|c|c|}
\hline $\begin{array}{l}\text { Jowett und Potter } \\
\text { Transact of the Chemical. } \\
\text { Soc. 19u2, } 1581\end{array}$ & aus & Chrysarobin & $190^{\circ}$ & - \\
\hline $\begin{array}{l}\text { Tschirch und Eijken. } \\
\text { Festsclirift Prof Vogl } 1904\end{array}$ & $n$ & Rheum palmatum & 1620 & mothoxylhaltig \\
\hline $\begin{array}{l}\text { Tschirch und Eijken . } \\
\text { Festschrift Prof. Vogl } 1904\end{array}$ & $n$ & " officinale & $172^{\circ}$ & $n$ \\
\hline $\begin{array}{l}\text { Tschirch und Eijken. } \\
\text { Festschrift Prof. Vogl } 1904\end{array}$ & , & n Wurzel & $172-174^{\circ}$ & - \\
\hline
\end{tabular}

Die Differenzen im Schmelzpunkt sind so groß, daß sich die Frage aufürängt, ob die aus verschiedenem Material dargestellten Chrysophansäuren wirklich miteiuander identisch sind, oder ob es sich vielleicht um isomere Verbindungen handelt. In diesem Sinne hat sich denn auch seinerzeit Tschirch ${ }^{1}$ ) geäußert. Später wies Hesse ${ }^{2}$ ) nach, daß in der Chrysophansäure fast immer durch Kochen mit Jodwasserstoffsäure Methoxyl nachweisbar ist, und nahm an, daß der wechselnde Sclumelzpunkt der Chrysophansäure auf einen wechselnden Gehalt an Chrysophansäuremethyläther zurückzuführen sei. Diese Annahme war um so berechtigter - namentlich für die aus Chrysarobin dargestellte Chrysophansäure --, als Jowett und P otter ${ }^{8}$ ) im käuflichen Chrysarobin einen Dichrysarobinmethyläther $\mathrm{C}_{31} \mathrm{H}_{26} \mathrm{O}_{7}$ nachgewiesen haben. Aehnlich wie aus dem Chrysarobin und dem Dichrysarobin bei der Oxydation Chrysophansäure entsteht, wird aus dem Dichrysarobinmethyläther zweifellos Clrysophansäuremethyläther gebildet werden. Es gelang $\mathrm{Hesse}$ durch wiederholtes Umkrystallisieren den Gehalt an Methoxyl, d. h. an Chrysophansäuremethyläther herabzudrücken und dadurch den Schmelzpunkt zu heben. Za demselben Resultate gelangten Tschirch und Heuberger ); auch sie fanden, daß darch öfteres Umkrystallisi-ren der Gehalt an Methoxyl zurückgeht und der Schmelzpunkt der Chrysophansäure steigt. Den høchsten, durch Umkrystallisieren erreichten Schmelzpunkt fand Hesse bei $188^{\circ}$; doch war auch in diesem Falle noch ein Gehalt von $0,36 \%$ Methoxyl nachzuweisen ${ }^{5}$ ). Zu einem noch höheren Schmelzpunkt $-190-192^{\circ}$ - gelangte Grandis $\left.{ }^{6}\right)$, indem er Chrysophansäure aus Chrysarobin, welche trotz wiederholtem Um-

1) Ber. d. d. pharm. Ges. 8 (1898), 189.

8) Ann. d. Chem. 309 (1899), 36.

$\left.{ }^{8}\right)$ Transact. of the Chem. Soc. 1902, 1582.

4) Die Beziehungen zwischen Methoxylgehalt und Schmelzpunkt der Chrysophansäure hat T s ch ir ch zusammengestellt (Festschrift Prof. Vogl 1904).

5) Ann. d. Chem. 309 (1899), 48.

o) Jahresber. d. Chem. 1892, 1654. 
krystallisieren einen zwischen $162-187^{\circ}$ schwankenden Schmelzpunkt zeigte, der Sublimation unterwarf. Ob diese bei $190-192^{\circ}$ schmelzende Chrysophansäure noch Methoxyl enthält, ist aus den Angaben Grandis - es war mir allerdings nur das Referat zugänglich - nicht ersichtlich; er selbst äußert die Ansicht, daß möglicherweise ein Isomeres der Chrysophansäure vorliegen könnte.

Um einen Anhaltspunkt zu gewinnen zur Entscheidung der Frage, ob die Chrysophansäuren verschiedener Provenienz miteinander identisch sind, haben T $\mathrm{s}$ (: $\mathrm{h}$ ir $\mathrm{ch}$ und $\mathrm{H}$ e u b e rge $\mathrm{r}^{\mathrm{l}}$ ) Versuche gemacht, auf andere Weise als durch Umkrystallisieren oder Sublimieren zu methoxylfreier Chrysophansäure von konstantem Schmelzpunkt zu gelangen. Durch Kochen mit Jodwasserstoffsäure ersetzten sie die Methoxylgruppen durch Hydroxyl und versuchten - jedoch obne befriedigende Resultate - das entstandene Chrysophansäurehydroanthron durch Oxydation in alkalischer Lösung, in Chrysophansäure überzuführen. Sie versuchten ferner, die methoxylhaltige Chrysophansäure zu methylieren, um eine vollständig methylierte Chrysophansäure zum Vergleich heranziehen zu können. Auch diese Versuche, die sowohl mit Halogenalkyl, als auch mit Dimethylsulfat ausgeführt wurden, blieben ohne Erfolg.

Ich habe die Versuche wièder anfgenommen und als Ausgangsmaterial Chrysophansäure benutzt, welche aus Chrysarobin durch Oxydation in alkalischer Losung dargestellt worden war und den Schmp. $175^{\circ}$ besaß. Durch Umkrystallisieren aus Benzol konnte das Produkt in zwei Fraktionen zerlegt werden, von denen die eine bei $186^{\circ}$, die zweite bei $165^{\circ}$ schmolz. Wiederholtes Umkrystallisieren der ersten Fraktion hatte auf den Schmelzpunkt keinen wesentlichen Einfluß. Die qualitative Prüfnng nach Zeisel ergab sowohl im Ausgangsmaterial als auch in den bei $165^{\circ}$ und bei $186^{\circ}$ schmelzenden Fraktionen erheblichen Gehalt an Methoxyl.

Methoxylfreie Chrysophansäure. Zur Verseifung der Methoxylgruppen wird Chrysophansăure in Benzol gelöst, in die Lösung zwei Teile fein gepulvertes Aluminiumchlorid eingetragen und das Gemisch, das sich bald blau färbt, 2-3 Stunden auf dem Wasserbade erhitzt. Das Benzol wird hierauf abgezogen, der Rückstand mit stark verdünnter Salzsäure versetzt, filtriert und ausgewaschen. Zur Entfernung harzartiger Produkte, die ungeløst zurückbleiben, zieht man den Filterinhalt mit verdünnter Natronlauge aus und fällt die tief rot gefärbte alkalische Lösung mit Salzsäure. Der Niederscblag wird nach dem Auswaschen und Trocknen in Benzol gelöst und die Lơsung mit Petroläther versetzt. Es entsteht dadurch ein roter, 1) Arch. d. Pharm. 1902, 605. 
voluminöser Niederschlag und aus dem Filtrate krystallisiert nach dem Einengen Chrysophansäure vom Schmp. 1930. Die weitere Reinigung geschieht am besten über das Acetat, welches dann durch längeres Kochen mit verdünnter Natronlauge verseift wird. Die Chrysophansäure fällt beim Ansäuern der filtrierten, tiefroten Lösung als voluminöser, gelber Niederschlag aus. Aus Benzol krystallisiert sie in goldglänzenden, braungelben Blättchen vom Schmp. 196. Durch Umkrystallisieren aus Benzol oder aus Alkohol blieb der Schmelzpunkt unverändert. Bei der Prüfung nach $Z$ eisel konnte auch nach zweistündigem Kochen mit Jodwasserstoffsäure kein Methoxyl mehr nachgewiesen werden.

Die Analyse der bei 1200 getrockneten Substanz ergab aus 0,2715 g $0,7100 \mathrm{CO}_{2}$ und $0,1070 \mathrm{H}_{2} \mathrm{O}$.

Berechnet für $\mathrm{C}_{14} \mathrm{H}_{5} \mathrm{O}_{2}\left(\mathrm{CH}_{8}\right)(\mathrm{OH})_{2}$ :

$$
\text { C } 70,87
$$

H $\quad 3,94$

In den Löslichkeitsverhältnissen stimmt die methoxylfreie Chrysophansäure mit den Chrysophansäuren früherer Autoren ïberein, und auch in dem Verhalten gegen Kalk- und Barytwasser ist kein Unterschied zu bemerken. Dagegen ist die Farbe erheblich dunkler als diejenige z. B. der Chrysophansäure vom Schmp. $186^{\circ}$.

Acetylchrysophansäure. Durch kurzes Kochen der Chrysophansäure vom Schmp. 196 ${ }^{7}$ mit Essigsäureanhydrid und essigsaurem Natrium entsteht das Acetat, welches nach dem Umkrystallisieren aus Essigsäure, Benzol oder Alkohol in blaßgelben Blättchen erhalten wird. Der Schmelzpunkt, den Liebermann bei $200^{\circ}$ und bei 202 bis $204^{\circ}$, Grandis bei $198-203^{\circ}$, und Jowett und Potter bei $203^{\circ}$ fanden, konnte durch öfteres Umkrystallisieren aus Benzol auf $208^{\circ}$ gebracht werden. In verdünnter Natronlauge löst sich das Acetat nur allmählich mit roter Farbe; es ist deshalb zar rollständigen Verseifung längeres Erwärmen notwendig.

Chrysophansäuremethyläther. Außer Tschirch und Heuberger haben sich auch schon Jowett und Pot te $\mathrm{r}^{1}$ ) bemüht, die Chrysophansäure zu methylieren. Ihre Versuche, die sie mit Methyljodid ansführten, blieben ebenfalls nhne Erfolg. Die Methyläther der Chrysophansäure lassen sich jedoch leicht darstellen, wenn man rohe Chrysophansäure in Kalilange löst und mit einem Ueberschaß von Dimethylsulfat einige Zeit schüttelt. Es scheidet sich ein Gemisch ab von unveränderter Chrysophansäure, deren Mono- und Dimethyläther, und dem Aether einer Substanz, deren Untersuchung noch nicht abgeschlossen ist. Zur Trennung dieses Gemisches wird die aus-

1) Transact. of the Chem. Soc. 1902, 1581. 
geschiedene Masse mit verdünnter Natronlange solange ausgekocht, bis die jeweilen abfiltrierte Flüssigkeit kaum noch rot gef ärbt ist. Aus der intensiv rot gef ärbten Lange scheidet sich nach einiger Zeit der Monomethyläther in langen, zu Klumpen verfilzten Nadeln aus. Die Abscheidung des Chrysophansäuremonomethyläthers aus der Lösung in Natronlauge geht, wenn die Lösung nicht konzentriert ist, ziemlich langsam vor sich. Man kann die Gewinnung des Aethers dadurch beschleunigen, daß man in die alkalische Lösung Kohlensäure einleitet. Der Niederschlag, der sich nach kurzer Zeit bildet, wird ansgewaschen and durch Kochen in verdünnter Natronlauge gelöst. Beim Erkalten scheidet sich der Monomethyläther aus. Die Fltissigkeit bleibt rot gefärbt und enthält neben unverănderter Chrysophansăure noch Mono. methyläther gelöst. Dieses, durch Zusatz von Säure ausgeschiedene Gemenge warde wieder zur Methylierung verwendet. Zur Reinigung wurde der Monomethyläther wiederholt aus verdünnter Essigsăure und aus Alkohol umkrystallisiert. Er bildet hell orange gefärbte, in konzentrierter Schwefelsăure mit gelbroter Farbe lösliche Nadeln, welche bei $204^{\circ}$ schmelzen. Auch das über das Acetat gereinigte Produkt zeigt denselben Schmelzpunkt.

Die Methoxylbestimmung nach Zeisel ergab aus 0,2315 $g$ Substanz 0,1946 Ag J.
Berechnet für $\mathrm{C}_{14} \mathrm{H}_{6} \mathrm{O}_{2} \mathrm{CH}_{8}(\mathrm{OH})\left(\mathrm{OCH}_{8}\right)$ : $\mathrm{CH}_{8} \mathrm{O} \quad 11,57$
Gefunden: 11,09 .

Acetylmonomethylchrysophansäure. Der Monomethylather der Chysophansäure wird durch Kochen mit Essigsäureanhydrid und Natriumacetat leicht acetyliert. Aus Essigsäure und hieranf aus Alkohol krystallisiert, bildet die Acetylverbindung zitronengelbe Nadeln vom Schmp. 204-2050. Dieser Schmelzpunkt, der auffallenderweise mit demjenigen der nicht acetylierten Verbindung zusammenfällt, bleibt, auch nach mehrmaligem Umkrystallisieren konstant. Durch Kochen mit alkoholischer Natronlange wird die Acetylmonomethylchrysophansăure verseift, der zurückgebildete Chysophansäuremonomethyläther schmilzt bei $204^{\circ}$.

Chrysophansäuredimethyläther. Beim Auskochen des durch die Methylierung gewonnenen Rohproduktes mit verdünnter Natronlauge, bleibt der Chrysophansäuredimethyläther ungelöst zurück. Er ist, wie sich bei den Versuchen zur Reinigung herausstellte, mit einer Substanz gemengt, von welcher er ziemlich schwierig zu trennen ist. Am besten gelingt die Reinigung und Trennung in folgender Weise. Der in Natronlauge unlősliche Rtickstand wird in Essigsăure gelöst, die Lösung mit Wasser bis zur beginnenden Trübung versetzt und einige Zeit mit Blutkohle gekocht. Aus dem Filtrat scheiden 
sich beim Erkalten Krystalle ab, welche, wenn sie in derselben Weise mehrmals umkrystallisiert werden, schließlich gelbe Farbe besitzen. Mit der Lupe ist deutlich zn erkennen, daß die Krystallausscheidung nicht einheitlich ist, sondern aus feinen, heller gefärbten und derben, dunkler gefärbten Nadeln besteht. Um dieses Gemisch zu trennen wurden die verschiedensten Versuche gemacht, die aber alle nicht befriedigende Resultate gaben. Am schnellsten gelingt die Trennung, wenn man das Krystallgemisch in einer Mischung von 70 Teilen $96 \%$ igem Alkohol und 30 Teilen Wasser durch Erwärmen löst; beim Erkalten der Lösung bleibt der größere Teil des Chrysophansäuredimethyläthers gelöst, während sich die begleitende Substanz, allerdings immer noch mit Chrysophansăareäther gemischt, ausscheidet. Indem man den ausgeschiedenen Anteil immer wieder in der Mischung von Alkohol und Wasser auflöst und sich wieder abscheiden läßt, gelingt es die beiden Substanzen von einander zu trennen. Aus den alkoholischen Lösungen wird der Chrysophansäuredimethyläther durch Wasserzusatz oder durch Eindampfen gewonnen und durch mehrmaliges Krystallisieren aus Essigsäure oder verdünntem Alkohol gereinigt. Er krystallisiert in ziemlich derben, gelborangefarbenen Nadeln und unterscheidet sich schon dadurch von der ihn begleitenden Substanz, welche unter denselben Bedingungen in langen, haarfeinen, wie Watte verfilzten Nadeln krystallisiert. Er löst sich leicht in Eisessig, Alkohol, Aceton, Essigăther, Chloroform, Benzol und Toluol, sowie in einer wässerigen Lösung von Pyridin, dagegen nur sehr wenig in heißem Wasser, Aether oder Petroläther. Aus der alkoholischen Lösung wird er durch Zusatz von Petroläther zum größten Teile ausgeschieden. In konzentrierter Schwefelsäure löst sich der Chrysophansäuredimethyläther mit roter Farbe; der Schmelzpunkt liegt bei $195^{\circ}$.

Zur Analyse wurde der Aether bei $120^{\circ}$ getrocknet.

$0,2344 \mathrm{~g}$ Substanz gaben $0,6215 \mathrm{CO}_{2}$ und $0,1115 \mathrm{H}_{2} \mathrm{O}$.

Berechnet für $\mathrm{C}_{14} \mathrm{H}_{5} \mathrm{O}_{2} \mathrm{CH}_{8}\left(\mathrm{OCH}_{8}\right)_{2}$ :

$$
\begin{array}{ccc}
\mathrm{C} & 72,31 & \mathbf{7 2 , 3 1} \\
\mathrm{H} & \mathbf{4 , 9 9} & \mathbf{5 , 2 8} .
\end{array}
$$

Bei den Versuchen, den Gehalt an Methoxyl nach Zeisel zu bestimmen, zeigte sich, daß die Substanz eine über der Jodwasserstoffsäure schwimmende, zusammenhängende Decke bildet und dadurch der Einwirkung der Jodwasserstoffsäure nicht ganz zugänglich ist. Der Gehalt an Methoxyl fel daher zo niedrig aus. Ein besseres Resultat wurde erzielt dadurch, daß nach dem Vorschlage von Herzig ${ }^{1}$ ) ein Zusatz von ca. $6 \%$ Essigsäureanhydrid gemacht wurde.

1) Monatsh. f. Chem. 9 (1898), 544. 
0,1940 g Substanz gaben 0,3227 Ag J.

Berechnet für $\mathrm{C}_{14} \mathrm{H}_{5} \mathrm{O}_{2} \mathrm{CH}_{3}\left(\mathrm{OCH}_{8}\right)_{2}$ :

$\mathrm{OCH}_{8} 22$

Gefunden:

21,95 .

Der Chrysophansäuredimethyläther läßt sich aus dem Monomethyläther durch Methylieren darstellen. Za diesem Zwecke löst man den Monomethylather in heißer Natronlauge und setzt unter anhaltendem Schütteln Dimethylsulfat im Ueberschusse zu. Die Ausbeute an Dimethyläther ist jedoch schlecht. Die Trennung des Dimethyläthers vom unveränderten Monometbyläther geschieht durch Auskochen mit verdünnter Natronlauge. Der Schmelzpunkt des auf diese Weise dargestellten Dimethyläthers liegt, nach einmaliger Krystallisation, bei $194,5-195^{\circ}$.

$\mathrm{Da}$ im allgemeinen der Schmelzpunkt durch Ersatz des Hydroxylwasserstoffs durch Acyl oder Alkyl erniedrigt wird, ist das Verhalten der Chrysophansäure bei der Acylierung und Alkylierung auffällig. Durch A cetylierung (wie auch durch Benzoylierung) wird der Schmelzpunkt gehoben und der Ersatz der beiden Hydroxylwasserstoffatome durch Methyl hat kaum eine Verănderung des Schmelzpunktes zar Folge. Die beiden Methyläther unter sich folgen dagegen der Regel, indem die Einführung einer zweiten Methylgruppe in den Monomethyläther den Schmelzpunkt von $204^{\circ}$ auf $195^{\circ}$ erniedrigt. Auffallig ist aber wieder, daß der Schmelzpunkt sich nicht verändert, wenn in den Monomethyläther an Stelle einer zweiten Methylgruppe, eine Acetylgruppe eingef ührt wird.

Dieses auffällige Verhalten gab Veranlassung zu Versuchen, aus dem Dimethyläther, dessen Schmelzpunkt mit demjenigen der methoxylfreien Chrysophansäure fast ganz übereinstimmt, durch Verseifen der Methoxylgruppen wieder za der Chrysophansäure za gelangen.

Versuche, den Aether durch einstündiges Erhitzen mit konzentrierter Schwefelsăure auf $100^{\circ} \mathrm{zu}$ verseifen, hatten nicht befriedigenden Erfolg. Die Verseifung läßt sich dagegen mit Aluminiumchlorid recht gut durchführen.

Der Dimethyläther wird in Benzol gelöst, mit fein gepulvertem Aluminiumchlorid versetzt und auf dem Wasserbade zum Sieden erhitzt. Das Gemisch färbt sich dabei nach wenigen Sekunden intensiv blau. Nach $1 \frac{1}{2}-2$ stündigem Erhitzen wird das Benzol abdestilliert, der Rückstand mit salzsäurehaltigem Wasser versetzt und filtriert. Die auf dem Filter befindliche Masse wird, nach dem Auswaschen mit verdünnter Natronlauge ausgezogen und die rote Lösung mit Salzsăure gefallt. Um allfällig, durch partielle Verseifung gebildeten Monomethyläther za entfernen löst man den Niederschlag kalt in verdünnter Natronlange, fäilt wieder mit Salzsäure und wiederbolt diese Operation zwei- bis dreimal. Schließlich wird der getrocknete Nieder- 
schlag acetyliert und das Acetat aus Alkohol umkrystallisiert. Nach zweimaligem Krystallisieren lag der Schmelzpunkt des Acetates bei $203^{\circ}$. Aus Mangel an Material muliten weitere Krystallisationen, die den Schmelzpunkt sicher noch gehohen hätten, unterbleiben. Die aus dem Acetat durch Verseifen mit Alkali gewonnene Chrysophansäure wurde einmal aus Alkohol unter Zusatz von etwas Wasser krystallisiert. Der Schmelzpunkt lag bei $195-196^{\circ}$, stimmt aber mit demjenigen der methoxylfreien Chrysophansäure überein.

Wie schon erwähnt, führte Hesse den schwankenden Schmelzpunkt der Chrysophansäure zurïck auf einen Gehalt an Chrysophansäuremetbyläther. Wie Versuche zeigten, wird in der Tat der Schmelzpunkt der methoxylfreien Chrysophansäure durch Zusatz der Methyläther herabgedrückt. Ein Gemisch von ungefähr gleichen Teilen Chrysophansăure (Schmp. 196 ${ }^{\circ}$ ) und Chrysophansäuredimethyläther (Schmp. 195 ) schmilzt bei 163-164 ${ }^{\circ}$; ein Gemisch von Chrysophansäure und dem Monometbyläther (Schmp. 204\%) besitzt den Schmp. 1650. Gegen die Annahme Hesse's spricht der Umstand, daß Chrysophansäuredimethyläther in Natronlauge unlöslich, und der Monomethyläther schwer lösilich ist. Eine Chrysophansäure, welche erhebliche Beimeng ungen der Aether, und namentlich des Dimethyläthers, enthält, sollte daher in Natronlauge nicht vollständig löslich sein. Tatsächlich ist aber methoxylhaltige Chrysophansäure, auch wenn sie einen ganz niedrigen Schmelzpunkt besitzt, in Natronlauge vollständig löslich.

Es scheint nun, daß die niedrig schmelzende Chrysophansäure, wie sie zu den vorstehenden Versuchen verwendet wurde, noch eine Substanz enthält, welche bei der Behandlung mit Dimethylsulfat ebenfalls methyliert wird und welche, wie bereits angedeutet wurde, nur schwierig von dem Chrysophansäuredimethyläther zu trennen ist. Diese Substanz krystallisierte in hellgelben, langen, haarfeinen, biegsamen Nadeln vom Schmp. 224\%. Bei der Prüfung nach $\mathrm{Z}$ eisel wurden Werte gefunden, welche auf das Vorhandensein von drei Methoxylgruppen schließen lassen, und es liegt nahe, die Substanz als Trimethyläther eines Trioxymethylanthrachinons aufzufassen. Bestätigt sich diese Vermutung, so müßte man annehmen, daß die Chrysophansäure - wenigstens diejenige aus Chrysarobin - von dem Mono- oder Dimethyläther dieser Substanz begleitet wird, und daß die Differenzen in den Schmelzpunkten auf diese Beimengung zurückzuftibren sind. Bei der Darstellung der methoxylfreien Chrysophansäure mittelst Aluminium. chlorid ist sie wahrscheinlich durch den Zusatz von Petroläther entfernt worden. Mit der Untersuchung dieser Substanz, sowie mit dem Studium der Chrysophausäureäther bin ich noch beschäftigt. 